\title{
Establishment of a Novel CD34+ Hematopoietic Stem Cell-derived Xenograft Model of Hyperleukocytic Acute Myeloid Leukemia
}

Yanxia Jin

Wuhan University https://orcid.org/0000-0002-8905-7315

Ziyi Luo

Wuhan University

Linlu Ma

Wuhan University

Yanling Chen

Wuhan University

Yujia Zheng

Wuhan University

Yuxing Liang

Wuhan University

Xiqin Tong

Wuhan University

Qian Yang

Wuhan University

Balu Wu

Wuhan University

Sanyun Wu

Wuhan University

Hui Shen

Wuhan University

Li Liu

Wuhan University

Yin Liu

Wuhan University

Tian Yang

Wuhan University

Fuling Zhou ( $\square$ zhoufuling@whu.edu.cn )

Wuhan University https://orcid.org/0000-0003-0982-0382 


\section{Short report}

Keywords: Hyperleukocytic acute myeloid leukemia, CD34+ hematopoietic stem cell, Patient-derived xenograft model, survival, B-NSG mice

Posted Date: October 5th, 2021

DOI: https://doi.org/10.21203/rs.3.rs-783481/v2

License: (c) (i) This work is licensed under a Creative Commons Attribution 4.0 International License.

Read Full License 


\section{Abstract}

\section{Background}

Hyperleukocytic acute myeloid leukemia (HLL) with high early mortality is difficult to therapy. The related research on HLL is still in its infancy, and the establishment of a patient derived xenograft (PDX) model of HLL has not been fully reported, especially the CD $34^{+}$hematopoietic stem cell-derived xenograft model of HLL.

\section{Methods}

Using the routine blood examination, smear analysis and bone marrow biopsy, flow cytometry, mutation analysis to evaluate the establishment of HLL model. And the correlation between the survival time in mice and in patients was analyzed by a linear regression model with variable selection "entered" with SPSS software.

\section{Results}

Our findings demonstrated that the leukocyte counts reached up to $37.35110^{9} / \mathrm{I}$, and the immunophenotypes of $\mathrm{hCD} 45^{+}, \mathrm{hCD} 15^{+}$and $\mathrm{hCD} 33^{+}$cells were detected in peripheral blood (PB) and bone marrow (BM) after inoculation with cells derived from PB for establishment of the HLL PDX model. The same results were demonstrated after inoculation with cells derived from BM of the patient. For the $\mathrm{CD} 34^{+}$hematopoietic stem cell derived xenograft model, the $\mathrm{CD} 34^{+}$hematopoietic stem cells more severely infiltrated the BM, liver and spleen. More importantly, human WT1 and NRAS mutants were detected in the liver, spleen and BM of the mouse. By performing a comparative analysis of multiple experiments, we found that mice receiving a higher irradiation dose of $2.5 \mathrm{~Gy}$ and higher injected cell counts derived from PB exhibited a shorter survival time. Furthermore, the constructed model mice injected with NRAS, DNMT3A, FLT3, or NMPM1 gene mutations had shorter survival times. The correlation analysis showed that the survival time in mice was significantly related with the survival status of the enrolled patients.

Conclusions

We successfully established a novel $\mathrm{CD} 34^{+}$hematopoietic stem cell-derived xenograft model of HLL, which shows great promise for mechanistic research, drug screening, individualized therapy, clinical efficacy assessment and precision medicine in HLL.

\section{Introduction}

Hyperleukocytic leukemia, a high-risk type, is commonly defined as peripheral blood leukocyte counts exceeding 50-100×10\%/I [1]; an abnormal increase in white blood cells (WBCs) causes an increase in blood viscosity and leads to leukocyte stasis [2] and leukemic organ infiltration in hyperleukocytic 
leukemia. Patients with hyperleukocytic acute myeloid leukemia (HLL) usually have higher mortality with severe life-threatening complications [3], for example, severe respiratory failure [4], disseminated intravascular coagulopathy and tumor lysis syndrome and $[1,5]$. The early mortality of hyperleukocytic leukemia patients is notable, with a mortality rate as high as $40 \%$ within a week [6,7]. Moreover, Routine clinical treatment is implemented to decrease the number of WBCs with leukapheresis, which is an effective therapeutic method to rapidly reduce leukocytes counts with a more rapid reduction in the leukemic cellular burden [5].

To study the pathogenesis of HLL and explore effective treatment, it is urgent to establish a suitable model of HLL. Traditional mouse models of leukemia are constructed by transplanting cell lines; however, immortalized cell lines may change their comparative functional phenotype and cannot represent the real state of disease [8]. The patient derived xenograft (PDX) model mainly transplants primary cells from fresh tumor cells or tissues of patients to immunodeficient mice, and is widely used to model of nonsmall cell lung cancer, bladder cancer, and triple-negative breast cancer [9-11] as well as acute myeloid leukemia (AML) [12]; the PDX model maintains the primary tumor microenvironment and original characteristics of patient in mice, making it more valuable in vivo than cell lines when constructing a leukemia model $[13,14]$. Therefore, the PDX model is a powerful research tool to explore the mechanism of tumor development and evaluate the potential therapeutic effects of disease. Primary patient-derived tumors require higher immunodeficiency levels for xenograft implantation, such as nonobese diabetic

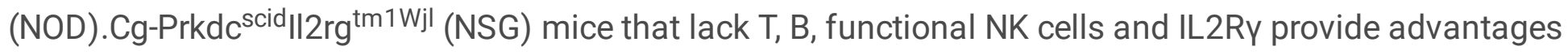
and are ideal hosts for in vivo engraftment studies regardless of their sensitivity to environmental status $[10,15]$.

At present, related research on HLL is still in its infancy, and establishment of a PDX model of HLL has not been reported, especially the construction of a $\mathrm{CD} 34^{+}$hematopoietic stem cell-derived xenograft model. In this study, we use the NOD.CB17-Prkdc ${ }^{\text {scid }} \| 2 \mathrm{rg}^{\text {tm1 } 1} /$ Bcgen (B-NSG) mice with severe immunodeficiency for HLL model establishment and evaluation.

\section{Methods}

- Patient-derived leukemia cell collection and culture

Human cells were collected from newly diagnosed HLL patients enrolled from Zhongnan Hospital of Wuhan University, and the information for the collected clinical samples is provided in Table 1. Patient diagnosis with AML was based on standard morphological and cytochemical examinations of peripheral blood and marrow smears according to the French-American-British (FAB) and World Health Organization (WHO) criteria. 
Table 1

The information of the collected clinical samples

\begin{tabular}{|c|c|c|c|c|}
\hline \multicolumn{2}{|l|}{ Information } & Patient \#1 & Patient \#2 & Patient \#3 \\
\hline \multicolumn{2}{|l|}{ Gender } & Male & Male & Female \\
\hline \multicolumn{2}{|l|}{ Age (year) } & 44 & 27 & 28 \\
\hline \multicolumn{2}{|l|}{ Clinical diagnosis } & AML-M5 & AML-M1 & AML-M1 \\
\hline \multirow{4}{*}{\multicolumn{2}{|c|}{$\begin{array}{l}\text { Detection of gene mutation } \\
\text { (PCR and sequencing) }\end{array}$}} & DNMT3A (+) & $W T 1(+)$ & $W T 1(+)$ \\
\hline & & NRAS (+) & NRAS (+) & SET-CAN $(+)$ \\
\hline & & NPM1 (+) & & \\
\hline & & FLT3-TKD (+) & & \\
\hline \multirow[t]{2}{*}{$\begin{array}{l}\text { Immunophenotype } \\
\text { (Flow cytometry) }\end{array}$} & $\begin{array}{l}\text { Abnormal cells } \\
\text { ratio }\end{array}$ & $58.50 \%$ & $74.6 \%$ & $71.8 \%$ \\
\hline & $\begin{array}{l}\text { Immunopheno- } \\
\text { type } \\
\text { expression }\end{array}$ & $\begin{array}{l}\text { CD36, HLA-DR, } \\
\text { CD11b, CD15, } \\
\text { CD38, CD33, CD64 }\end{array}$ & $\begin{array}{l}\text { CD34, CD33, } \\
\text { CD11b, CD7, } \\
\text { CD123, CD99, } \\
\text { CD71 }\end{array}$ & $\begin{array}{l}\text { CD7, CD34, CD33, } \\
\text { CD19, CD11b, } \\
\text { CD79a, CD38 }\end{array}$ \\
\hline
\end{tabular}

The HLL cells were collected using a Fresenius COM.TEC machine at Zhongnan Hospital; the procedures were described in our previous reports [16]. Peripheral blood mononuclear cells (PBMCs) were separated with a Ficoll kit according to the manufacturer's instructions (TBD, lot: LDS1075, Tianjin, China). The separated cells were maintained in RPMI 1640 media containing 10\% FBS (Gibco, Invitrogen, Carlsbad, CA, USA) with $100 \mathrm{U} / \mathrm{ml}$ penicillin and $100 \mu \mathrm{g} / \mathrm{ml}$ streptomycin at $37^{\circ} \mathrm{C}$ and $5 \% \mathrm{CO}_{2}$. Bone marrow-derived cells were collected from HLL patients during bone marrow biopsy and separated using a red blood cell lysis buffer.

- Isolation of $\mathrm{CD}_{3} 4^{+}$cells

$\mathrm{CD}_{4}{ }^{+}$cells were separated using the CD34 MicoBeads Kit (Miltenyi Biotec, Germany) according to the manufacturer's instructions. In brief, PBMCs from HLL patients (Patient \#2) with WT1 and NRAS mutations were magnetically labeled with CD34 microbeads for 30 min at $4{ }^{\circ} \mathrm{C}$, and then centrifuged at $300 \mathrm{~g}$ for $10 \mathrm{~min}$. The cell suspension was loaded onto a MACS ${ }^{\circledR}$ column with a MACS separator. The magnetically labeled $\mathrm{CD} 34^{+}$cell subpopulations were retained within the column, and the unlabeled cells were washed. After removing the column from the magnetic field, the magnetically retained $\mathrm{CD} 34^{+}$cells were eluted as the positively selected cell fraction.

- Animal experiments

Five- to six-week-old female B-NSG mice (NOD.CB17-Prkdc ${ }^{\text {scid }} \mid 2 \mathrm{rg}^{\mathrm{tm} 1} /$ Bcgen) were obtained from Beijing Biocytogen Co., Ltd. (permission number: SCXK 2016-0004) and maintained in a pathogen-free animal 
facility in laminar airflow cabinets with a $12 \mathrm{~h}$ light/12 $\mathrm{h}$ dark schedule. The animals were fed an autoclaved rodent diet ad libitum. All procedures followed the institutional and national guidelines for the care and use of laboratory animals.

For HLL PDX model establishment with PBMCs of patients, patient-derived xenografts (PDXs) were constructed as described below. On day 0, NOD-SCID-IL2ry ${ }^{\text {null }}(B-N S G)$ mice were treated with 0 Gy $(n=5)$ or $2.5 \mathrm{~Gy}(\mathrm{n}=5) \chi$-rays for total body irradiation and intravenously injected with harvested human HLL cells from Patient \#1 mixed with granulocyte colony-stimulating factor (G-CSF). Every mouse was injected with $1.5 \times 10^{7}$ cells within $24 \mathrm{~h}$ of irradiation.

For HLL PDX model establishment with BM-derived cells from patients, PDXs were constructed as described below. On day 0, B-NSG mice were treated with $0 \mathrm{~Gy}(\mathrm{n}=5)$ or $1.5 \mathrm{~Gy}(\mathrm{n}=6)$ X-rays for total body irradiation and intravenously injected with harvested BM-derived cells from a human HLL patient (Patient \#3) at day 1 . The mice in the $1.5 \mathrm{~Gy}$ irradiated group were injected with $2.0 \times 10^{6}$ cells within $24 \mathrm{~h}$ of irradiation, and the mice without irradiation were injected with $1.5 \times 10^{7}$ cells. Blood samples were collected at intervals to test whether the model was successfully constructed.

For the CD34 ${ }^{+}$hematopoietic stem cell-derived xenograft model of HLL, NOD-SCID-IL2ry ${ }^{\text {null }}$ (B-NSG) mice were treated with $1.5 \mathrm{~Gy} \chi$-rays for total body irradiation on day 0 and intravenously injected with isolated

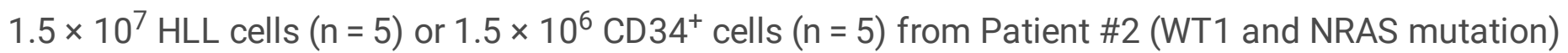
mixed with G-CSF within $24 \mathrm{~h}$ of irradiation.

On day 9, peripheral blood was collected through the tail vein in mice to validate whether these two models were successfully constructed. Routine blood tests were performed and body weights were determined at regular intervals. The mice were anesthetized with intraperitoneal (i.p.) injection of sodium pentobarbital administration at $120 \mathrm{mg} / \mathrm{kg}$, then the harvested blood, bone marrow and tissue samples were collected for further analysis. The experimental procedures were approved by Experimental Animal Ethics Committee of Wuhan University.

- Routine blood examination

Approximately $50 \mu \mathrm{l}$ of blood was used for routine blood examination with a Coulter STKS automated blood cell analyzer (Beckman Coulter, USA).

- Smear analysis and bone marrow biopsy

Blood smears and bone marrow smears were stained via Wright's staining and observed with a microscope under an oil immersion lens (OLYMPUS BX41) to measure the ratio of leukemia cells.

For the bone marrow biopsy, bone marrow collected from mouse thighs was fixed in formalin and embedded in paraffin for bone marrow examination. The sections were sliced using a slicing machine 
(Leica RM2016) and incubated with anti-CD34 (1:200, BD Biosciences). Finally, the images were observed with an OLYMPUS BX53 microscope.

- HE staining and immunohistochemistry

The tissues were fixed with formalin and embedded in paraffin to test the infiltration of leukemic cells by HE staining or immunohistochemistry. For IHC, the tissues were stained with antibodies against human anti-CD45 ${ }^{+}$(1:200, BD Biosciences) or human anti-CD34 ${ }^{+}$(1:200, BD Biosciences). The images were observed with an OLYMPUS BX41 microscope.

\section{Flow cytometry}

Harvested mouse peripheral blood and bone marrow were treated with RBC lysis buffer, HLL cells from Patient \#1 were stained with human anti-CD45-PerCPcy5.5, anti-CD15-FITC, and anti-CD33-PE antibodies (BD Biosciences), and HLL or CD34 ${ }^{+}$cells from Patient \#2 were stained with human anti-CD45PerCPcy5.5 and anti-CD34-APC antibodies (BD Biosciences). The stained cells were analyzed by flow cytometry (FACS Canto II, FACS Verse, and FACS Diva software, BD Biosciences), and data were analyzed with FlowJo Software.

- RNA extraction and quantitative real-time PCR (qPCR) detection

The collected fresh liver and spleen tissues from mice were treated and prepared as single cell suspensions. Then, total RNA from the liver, spleen and bone marrow was extracted with TRIzol reagent (Vazyme, Lot: R401-01). The cDNAs were obtained by Evo M-MLV reverse transcript kits (Accurate Biotechnology Co., Ltd, Lot: AG11705).

All qPCR reactions were performed with the SYBR® Premix Ex Taq ${ }^{\text {TM }}$ kit (Takara) for 40 cycles using a fluorescence quantitative PCR instrument (Applied Biosystems ABI 7500). The primer sequences were synthesized by Tsingke Biological Technology (Wuhan, China) and were as follows: (1) human WT1 gene forward (CAGGCTGCAATAAGAGATATTTTAAGCT) and reverse (GAAGTCACACTGGTATGGTTTCTCA), and (2) human reference gene for ABL gene forward (TGGAGATAACACTCTAAGCATAACTAAAGGT) and reverse (GATGTAGTTGCTTGGGACCCA). The positive rate for the WT1 gene in the mice was calculated.

- NRAS mutation analysis

DNA was extracted from the liver, spleen and bone marrow using a DNA Extraction System (TIANGEN, Lot: DP304). The PCR primer sequences for the NRAS gene were synthesized by Tsingke Biological Technology (Wuhan, China) and were as follows: forward (TAGCTAAGGATGGGGGTTGC) and reverse (ACTGGGCCTCACCTCTATGG). PCR was performed with $2 \times$ Hieff $^{\mathrm{TM}}$ PCR Master Mix (YEASEN, Lot: $\mathrm{H} 2001071$ ), and the products were sequenced by Tsingke Biological Technology (Wuhan, China) and analyzed by Chromas software (version 1.62). 


\section{Results}

- Construction of the HLL model with PBMCs from patients

The individual information of enrolled HLL patients were collected from the electronic patient records and provided in Supplementary Table 1. PBMCs were collected from HLL patients who underwent leukapheresis by using a Fresenius COM.TEC blood cell separator. The collected leukocytes were isolated by density gradient centrifugation and cultured. The cell viability of separated leukemic cells was still over $90 \%$ after culture for four days (Additional file: Figure S1).

Harvested HLL cells were intravenously injected into B-NSG mice to construct the HLL PDX model, and the integrated workflow strategy is shown in Fig. 1a. The body weights of mice in the $2.5 \mathrm{~Gy}$ irradiated group gradually decreased (Fig. 1b). The nonirradiated mice survived longer than the mice in the $2.5 \mathrm{~Gy}$ irradiated group, and the survival rate was $66.67 \%$ (Fig. 1c). On the 9th day, the immunophenotype ratios of hCD45+, hCD15 + and hCD33 + cells in the 2.5 Gy irradiated group were higher than that of nonirradiated group (Fig. 1d). This shows that the HLL model can be successfully established under these two conditions. Because of the rapid death and poor survival of the mice in the $2.5 \mathrm{~Gy}$ irradiated group, we performed related experiments on the nonirradiated group. Routine blood examination showed that WBC counts first increased and gradually decreased in the peripheral blood (PB) of B-NSG mice, and the maximum leukocyte count was above $37.35 \times 10^{9} / \mathrm{L}$. The red blood cell counts, platelet counts and hemoglobin content decreased gradually (Fig. 1e-h). Leukemic cells were found on the 9th, 21st and 25th days by blood smear analysis (Fig. 1i), and the immunophenotype ratio of hCD $45^{+}$cells increased up to 73.8\% gradually in PB (Fig. 1j). Many leukemic cells infiltrated the bone marrow (BM) on the 25th day and even caused erythrocyte lysis, which was observed on a bone marrow smear (Fig. 1k). The immunophenotype ratio of hCD $45^{+}$cells in bone marrow were $43.62 \%(9.5 \%-75.2 \%)$, the hCD $15^{+}$cells were $54.07 \%$ (3.3\%-79.9\%) and the hCD33 ${ }^{+}$cells were $48.4 \%$ (5.7\%-80.4\%) (Fig. 1I). In addition, the histopathological analysis indicated that a large number of human leukemic cells infiltrated the liver and spleen tissue of the mouse (Fig. 1m).

- Construction of the HLL model with BM-derived cells of patients

Furthermore, we collected the BM-derived cells of patients (Patient \#3) who underwent BM aspiration, and the separated BM-derived cells were injected into B-NSG mice. The body weights were worse and the survival was poorer in the 1.5 Gy group than the 0 Gy group (Fig. 2a, b). Cell morphology analysis showed that human leukemia cells were present in blood smears and detected in blood smear experiments (Fig. 2c, d). Furthermore, the immunophenotype ratios of human leukemia cells in the PB and BM of mice were increased (Fig. 2e, f).

- Successful construction of the $\mathrm{CD} 34^{+}$cell-derived xenograft model

To better explore the HLL model, we constructed a CD34 ${ }^{+}$hematopoietic stem cell-derived xenograft model of HLL. First, the workflow of model establishment is shown in Fig. 3a. At day 9, the 
immunophenotypes of hCD $45^{+}$and hCD $34^{+}$cells were detected at higher ratios in the $\mathrm{CD} 34^{+}$group with $\mathrm{PB}$, and leukemia cells were also detected (Fig. 3b, c), which suggested that the model was successfully established. The body weights of mice in the $\mathrm{CD} 34^{+}$group decreased faster due to the severe tumor load (Fig. 3d). In the CD $34^{+}$group, routine blood examination indicated changes, especially the WBC counts and PLT counts (Fig. 3e, f, g, h). On the 25th day, leukemic cells were found by blood smear analysis and flow cytometry (Fig. $3 \mathrm{i}, \mathrm{j}$ ), which were more obviously observed in $\mathrm{BM}$, and the immunophenotype ratios of $\mathrm{hCD} 45^{+}$and $\mathrm{hCD} 34^{+}$cells were slightly increased in the $\mathrm{CD} 34^{+}$group (Fig. $3 \mathrm{k}, \mathrm{I}$ ). Further analysis showed that $\mathrm{CD} 34^{+}$cells infiltrated into the BM, liver and spleen tissues on the 25th day, which indicated more obvious model construction using CD34+ hematopoietic stem cells (Fig. $3 \mathrm{~m}$ ). We also detected gene mutations in mice samples and found that the WT1 gene was positive in the liver, spleen and BM in mice (Fig. 3n). Moreover, NRAS mutations were found in the liver, spleen and BM in mice (Fig. 3o).

\section{- Summary of the constructed HLL models with B-NSG mice}

The HLL models were constructed for multiple repeated experiments, and the patient information, separated cell characteristics, irradiation doses, injection cell counts, and survival time are summarized in Fig. 4a. By comparison analysis, the mice treated with a higher irradiation dose of $2.5 \mathrm{~Gy}$ had a shorter survival time (Fig. $4 \mathrm{~b}$ ), and the more injection of cells derived from PB resulted in a shorter survival time (Fig. 4c). In the absence of irradiation, the constructed model mice injected with cells derived from PB had shorter survival times than the mice injected with cells derived from BM of patients; however, there were no significant differences in survival time between the $1.5 \mathrm{~Gy}$ irradiated model mice receiving cells derived from PB and BM (Fig. 4d). Furthermore, mutations of the NRAS, DNMT3A, FLT3, and NPM1 genes were also analyzed, which indicated that the model mice injected with the NRAS gene mutation had a shorter survival time than those injected with cells lacking the NRAS gene mutation, and the same results were obtained with the DNMT3A, FLT3, and NMPM1 gene mutations (Fig. 4e). In addition, correlation analysis showed that the survival time in mice had a significant correlation with the survival status of enrolled patients (Fig. 4f).

\section{Discussion}

In this study, we used B-NSG mice with an interleukin-2 receptor gamma chain (IL2RY) deletion and nearly complete absence of the murine immune system to construct an HLL model, to improve HLL cell engraftment and reduce graft versus host disease $[17,18]$. The above experimental results showed that we successfully established a CD34 ${ }^{+}$hematopoietic stem cell-derived xenograft model of human HLL in B-NSG mice. First, a large number of HLL cells could be detected in blood by transplanting cells into the mice, and leukemic cells were observed based on cell morphology in the peripheral blood and bone marrow of mice. Then, a higher ratio of the immunophenotype of leukemic cells was detected by flow cytometry, which was consistent with the immunophenotype of the patients. Finally, the leukemic cells were severely infiltrated into other tissues, and gene mutations were detected in infiltrating tissues in the CD34 ${ }^{+}$hematopoietic stem cell-derived xenograft model. 
According to previous reports, 1.5-3.5 Gy doses of $\chi$-ray radiation have been used before leukemia cell $[19-21]$ or $C D 34^{+}$cells $[22,23]$ transplantation into mice, but only a few studies did not use irradiation [24]; for example, Shafat et al. reported the successful construction of a primary AML model with nonirradiated NSG mice [25]. In this study, we explored the radiation dose in model construction. For the 2.5 Gy $x$-ray irradiated group, the body weight loss of mice was faster, and the survival time was shorter than that of the $0 \mathrm{~Gy}$ irradiated group, which indicated the $0 \mathrm{~Gy}$ irradiated mice could better used for drug research. The body weight of mice did not decrease much, and their body condition was good in the $0 \mathrm{~Gy}$ irradiated group; however, the mice in the 2.5 Gy group survived approximately 17 days and were in poor condition, dying easily, which is unfavorable for the study of drug efficacy or the mechanism of HLL development. Importantly, irradiation can briefly result in serious myelosuppression[26] and lead to an inability to clearly observe leukemic cells in blood smears and bone marrow smears, making it difficult to assess whether the model was successful. Moreover, myelosuppression seriously affects routine blood tests and cannot truly allow the evaluation of changes in WBCs $[27,28]$. Our results also showed that myelosuppression caused by irradiation could affect the survival time of mice and possibly lead to no significant difference in survival time between the $1.5 \mathrm{~Gy}$ irradiated model mice injected with cells derived from the PB and BM of patients.

In our previous reports on drug screening using a PDX model of HLL, B-NSG mice in the 2.5 Gy group survived beyond 20 days when injected with other HLL cells without malignant gene mutations [29], and B-NSG mice without irradiation survived beyond 31 days when injected with human AML cells separated from a hyperleukocytic AML-M5 patient with NPM1 and DNMT3A mutations [30]. In addition, the B-NSG mice with 1.5 Gy irradiation survived beyond 20 days when injected with human AML cells separated from a hyperleukocytic AML-M5 patient with $W T 1, D N M T 3 A$ and FLT3 mutations [31, 32]. The mutational status of AML cells is also a key factor in immunodeficient mice for the construction of a PDX model [33, 34]. In this study, we found that the constructed model mice injected with NRAS gene mutations had shorter survival times than the injected with cells lacking the NRASgene mutations, and the same results were observed for DNMT3A, FLT3, and NMPM1 gene mutations. The survival time in mice had significant correlation with the survival status of enrolled patients. Therefore, our results suggest that using B-NSG mice for HLL PDX construction is dependent on the use of HLL cells from patients who have malignant gene mutations and poor prognosis and is also related to the injected cell counts and the irradiation dosage in mice.

To establish a $\mathrm{CD} 34^{+}$hematopoietic stem cell-derived xenograft model with human hyperleukocytic AML cells, we found that the use of nonirradiated B-NSG mice injected with CD34 ${ }^{+}$cells from HLL patients without malignant gene mutations was not successful, and until the 50th day, leukemic cells were not found in blood smears or bone morrow smears. In this study, B-NSG mice were treated with 1.5 Gy X-ray irradiation and injected with $\mathrm{CD} 34^{+}$cells derived from a HLL patient with $W T 1$ and NRAS mutations, two mutations that frequently occur in AML [35]. The CD34+ cell-derived PDX model of HLL was established successfully, which also indicated that B-NSG mice with low-dose radiation were better used for model construction based on injection of cells with moderate malignant mutations.

Page $10 / 22$ 
However, the HLL model still has some limitations; for example, HLL cells from patients show difficulty proliferating in vitro, and the mice do not present full clinical characteristics, such as certain immunophenotypes, so the PDX model of HLL is still not standardized and requires further investigation, and we are still unable to replicate the complex immune microenvironments in immunodeficiency mice [36], so it is difficult to explore the complex heterogeneity of HLL and the interaction and progression between HLL cells and their microenvironment [14,37]. Further related experiments will be investigated. Even so, the HLL mouse model is still of great value in the study of the pathogenesis and drug efficacy.

\section{Conclusion}

In conclusion, we transplanted hyperleukemic leukemia cells into severe immunodeficient B-NSG mice, which retained most of the original characteristics in the B-NSG mice, and more accurately simulate the characteristics of tumor growth in clinical patients. we assessed the construction methods used for HLL model, optimized in vivo model establishment, and successfully established the CD $34^{+}$hematopoietic stem cell-derived xenograft model of HLL in B-NSG mice, and provide a meaningful model for mechanistic research, drug screening, individualized therapy, clinical efficacy assessment and precision medicine in HLL.

\section{Abbreviations}

HLL: Hyperleukocytic acute myeloid leukemia; PDX: patient derived xenograft; WBCs: white blood cells; AML: acute myeloid leukemia; PB: peripheral blood; BM: bone marrow.

\section{Declarations}

\section{Ethics approval and consent to participate}

The study was carried out followed the Declaration of Helsinki and approved by the Research Ethics Committee of Zhongnan Hospital at Wuhan University (license number: 2017048). Informed written consent was obtained from the HLL patients.

\section{Consent for publication}

Not applicable.

\section{Data Availability Statement}

All data are available in the main text or the Supplementary materials.

\section{Competing interests}

The authors declare that they have no competing interests. 
Funding

This article was supported by the Natural Science Foundation of China (NSFC) programme (No. 81770179; No. 320000908).

\section{Author's contribution}

FZ and YJ conceived the study and provided the project direction. YJ designed, executed and performed the experiments, analyzed the data and wrote the manuscript until the final submission version. $Z L, L M$, YC, YZ, YL, XT, QY, BW, YL and TY helped to collect the patients' information, separate the cells from patients, executed and interpreted the animal experiments. QY performed the MTT assays. SW carried out the qPCR. HS assisted and supervised the smear analysis. LL assisted and supervised the flow cytometry. FZ revised the manuscript. All authors read and approved the final manuscript.

\section{Acknowledgements}

Not applicable.

\section{References}

1. Bertoli S, Picard M, Berard E, Griessinger E, Larrue C, Mouchel PL, et al. Dexamethasone in hyperleukocytic acute myeloid leukemia. Haematologica. 2018;103(6):988-98.

2. Giammarco S, Chiusolo P, Piccirillo N, Di Giovanni A, Metafuni E, Laurenti L, et al. Hyperleukocytosis and leukostasis: management of a medical emergency. Expert Rev Hematol. 2017;10(2):147-54.

3. Feng S, Zhou L, Zhang X, Tang B, Zhu X, Liu H, et al. Impact Of ELN Risk Stratification, Induction Chemotherapy Regimens And Hematopoietic Stem Cell Transplantation On Outcomes In Hyperleukocytic Acute Myeloid Leukemia With Initial White Blood Cell Count More Than 100 x 10(9)/L. Cancer Manag Res. 2019;11:9495-503.

4. Bretz CA, Savage SR, Capozzi ME, Suarez S, Penn JS. NFAT isoforms play distinct roles in TNFalphainduced retinal leukostasis. Sci Rep. 2015;5:14963.

5. Rollig C, Ehninger G. How I treat hyperleukocytosis in acute myeloid leukemia. Blood. 2015;125(21):3246-52.

6. Ali AM, Mirrakhimov AE, Abboud CN, Cashen AF. Leukostasis in adult acute hyperleukocytic leukemia: a clinician's digest. Hematol Oncol. 2016;34(2):69-78.

7. Stefanski M, Jamis-Dow C, Bayerl M, Desai RJ, Claxton DF, Van de Louw A. Chest radiographic and CT findings in hyperleukocytic acute myeloid leukemia: A retrospective cohort study of 73 patients. Medicine (Baltimore). 2016;95(44):e5285. 
8. Pan C, Kumar C, Bohl S, Klingmueller U, Mann M. Comparative proteomic phenotyping of cell lines and primary cells to assess preservation of cell type-specific functions. Mol Cell Proteomics. 2009;8(3):443-50.

9. Chaturvedi A, Herbst L, Pusch S, Klett L, Goparaju R, Stichel D, et al. Pan-mutant-IDH1 inhibitor BAY1436032 is highly effective against human IDH1 mutant acute myeloid leukemia in vivo. Leukemia. 2017;31(10):2020-8.

10. Okada S, Vaeteewoottacharn K, Kariya R. Application of Highly Immunocompromised Mice for the Establishment of Patient-Derived Xenograft (PDX) Models. Cells. 2019;8(8).

11. Wang M, Yao LC, Cheng M, Cai D, Martinek J, Pan CX, et al. Humanized mice in studying efficacy and mechanisms of PD-1-targeted cancer immunotherapy. FASEB J. 2018;32(3):1537-49.

12. Griessinger E, Andreeff M. NSG-S mice for acute myeloid leukemia, yes. For myelodysplastic syndrome, no. Haematologica. 2018;103(6):921-3.

13. Vick B, Rothenberg M, Sandhofer N, Carlet M, Finkenzeller C, Krupka C, et al. An advanced preclinical mouse model for acute myeloid leukemia using patients' cells of various genetic subgroups and in vivo bioluminescence imaging. PLoS One. 2015;10(3):e0120925.

14. Almosailleakh M, Schwaller J. Murine Models of Acute Myeloid Leukaemia. Int J Mol Sci. 2019;20(2).

15. Maletzki C, Bock S, Fruh P, Macius K, Witt A, Prall F, et al. NSG mice as hosts for oncological precision medicine. Lab Invest. 2020;100(1):27-37.

16. Jin Y, Guo S, Cui Q, Chen S, Liu X, Wei Y, et al. A hospital based retrospective study of factors influencing therapeutic leukapheresis in patients presenting with hyperleukocytic leukaemia. Sci Rep. 2018;8(1):294.

17. Shultz LD, Lyons BL, Burzenski LM, Gott B, Chen X, Chaleff S, et al. Human lymphoid and myeloid cell development in NOD/LtSz-scid IL2R gamma null mice engrafted with mobilized human hemopoietic stem cells. J Immunol. 2005;174(10):6477-89.

18. Covassin L, Laning J, Abdi R, Langevin DL, Phillips NE, Shultz LD, et al. Human peripheral blood CD4 T cell-engrafted non-obese diabetic-scid IL2rgamma(null) H2-Ab1 (tm1Gru) Tg (human leucocyte antigen D-related 4) mice: a mouse model of human allogeneic graft-versus-host disease. Clin Exp Immunol. 2011;166(2):269-80.

19. Busfield SJ, Biondo M, Wong M, Ramshaw HS, Lee EM, Ghosh S, et al. Targeting of acute myeloid leukemia in vitro and in vivo with an anti-CD123 mAb engineered for optimal ADCC. Leukemia. 2014;28(11):2213-21. 
20. Martelli MF, Di lanni M, Ruggeri L, Falzetti F, Carotti A, Terenzi A, et al. HLA-haploidentical transplantation with regulatory and conventional T-cell adoptive immunotherapy prevents acute leukemia relapse. Blood. 2014;124(4):638-44.

21. Dong S, Chen J. SUMOylation of SPRDM16 promotes the progression of acute myeloid leukemia. BMC Cancer. 2015;15(1):893-.

22. Demmerath $E$, Bohler $S$, Kunze $M$, Erlacher M. In vitro and in vivo evaluation of possible pro-survival activities of PGE2, EGF, TPO and FLT3L on human hematopoiesis. Haematologica. 2019;104(4):669-77.

23. He X, Zhu Y, Lin Y, Li M, Du J, Dong H, et al. PRMT1-mediated FLT3 arginine methylation promotes maintenance of FLT3-ITD+ acute myeloid leukemia. Blood. 2019;134(6):548-60.

24. Gopalakrishnapillai A, Kolb EA, Dhanan P, Bojja AS, Mason RW, Corao D, et al. Generation of Pediatric Leukemia Xenograft Models in NSG-B2m Mice: Comparison with NOD/SCID Mice. Front Oncol. 2016;6:162.

25. Shafat MS, Oellerich T, Mohr S, Robinson SD, Edwards DR, Marlein CR, et al. Leukemic blasts program bone marrow adipocytes to generate a protumoral microenvironment. Blood. 2017;129(10):1320-32.

26. Lu L, Wang YY, Zhang JL, Li DG, Meng AM. p38 MAPK Inhibitor Insufficiently Attenuates HSC Senescence Administered Long-Term after 6 Gy Total Body Irradiation in Mice. Int J Mol Sci. 2016;17(6).

27. Sun C, Yang J, Pan L, Guo N, Li B, Yao J, et al. Improvement of icaritin on hematopoietic function in cyclophosphamide-induced myelosuppression mice. Immunopharmacol Immunotoxicol. 2018;40(1):2534.

28. Chisaki Y, Terada T, Yano Y. Population Pharmacodynamic Model for Bayesian Prediction of Myelosuppression Profiles Based on Routine Clinical Data after Gemcitabine and Carboplatin Treatment. Pharmacology. 2016;98(5-6):284-93.

29. Jin $Y$, Yang Q, Liang L, Ding L, Liang Y, Zhang D, et al. Compound kushen injection suppresses human acute myeloid leukaemia by regulating the Prdxs/ROS/Trx1 signalling pathway. J Exp Clin Cancer Res. 2018;37(1):277.

30. Jin Y, Cai L, Yang Q, Luo Z, Liang L, Liang Y, et al. Anti-leukemia activities of selenium nanoparticles embedded in nanotube consisted of triple-helix beta-d-glucan. Carbohydr Polym. 2020;240:116329.

31. Zhang D, Luo Z, Jin Y, Chen Y, Yang T, Yang Q, et al. Azelaic Acid Exerts Antileukemia Effects against Acute Myeloid Leukemia by Regulating the Prdxs/ROS Signaling Pathway. Oxid Med Cell Longev. 2020;2020:1295984. 
32. Zhang $D$, Liu Y, Luo Z, Chen $Y, X u A$, Liang $Y$, et al. The novel thioredoxin reductase inhibitor A-Z2 triggers intrinsic apoptosis and shows efficacy in the treatment of acute myeloid leukemia. Free Radic Biol Med. 2020;146:275-86.

33. Culen M, Kosarova Z, Jeziskova I, Folta A, Chovancova J, Loja T, et al. The influence of mutational status and biological characteristics of acute myeloid leukemia on xenotransplantation outcomes in NOD SCID gamma mice. J Cancer Res Clin Oncol. 2018;144(7):1239-51.

34. Paczulla AM, Dirnhofer S, Konantz M, Medinger M, Salih HR, Rothfelder K, et al. Long-term observation reveals high-frequency engraftment of human acute myeloid leukemia in immunodeficient mice. Haematologica. 2017;102(5):854-64.

35. Kunimoto H, Meydan C, Nazir A, Whitfield J, Shank K, Rapaport F, et al. Cooperative Epigenetic Remodeling by TET2 Loss and NRAS Mutation Drives Myeloid Transformation and MEK Inhibitor Sensitivity. Cancer Cell. 2018;33(1):44-59 e8.

36. Vago L, Gojo I. Immune escape and immunotherapy of acute myeloid leukemia. J Clin Invest. 2020;130(4):1552-64.

37. Cook GJ, Pardee TS. Animal models of leukemia: any closer to the real thing? Cancer Metastasis Rev. 2013;32(1-2):63-76.

\section{Tables}

Supplementary Table 1 is not available with this version.

\section{Figures}


a
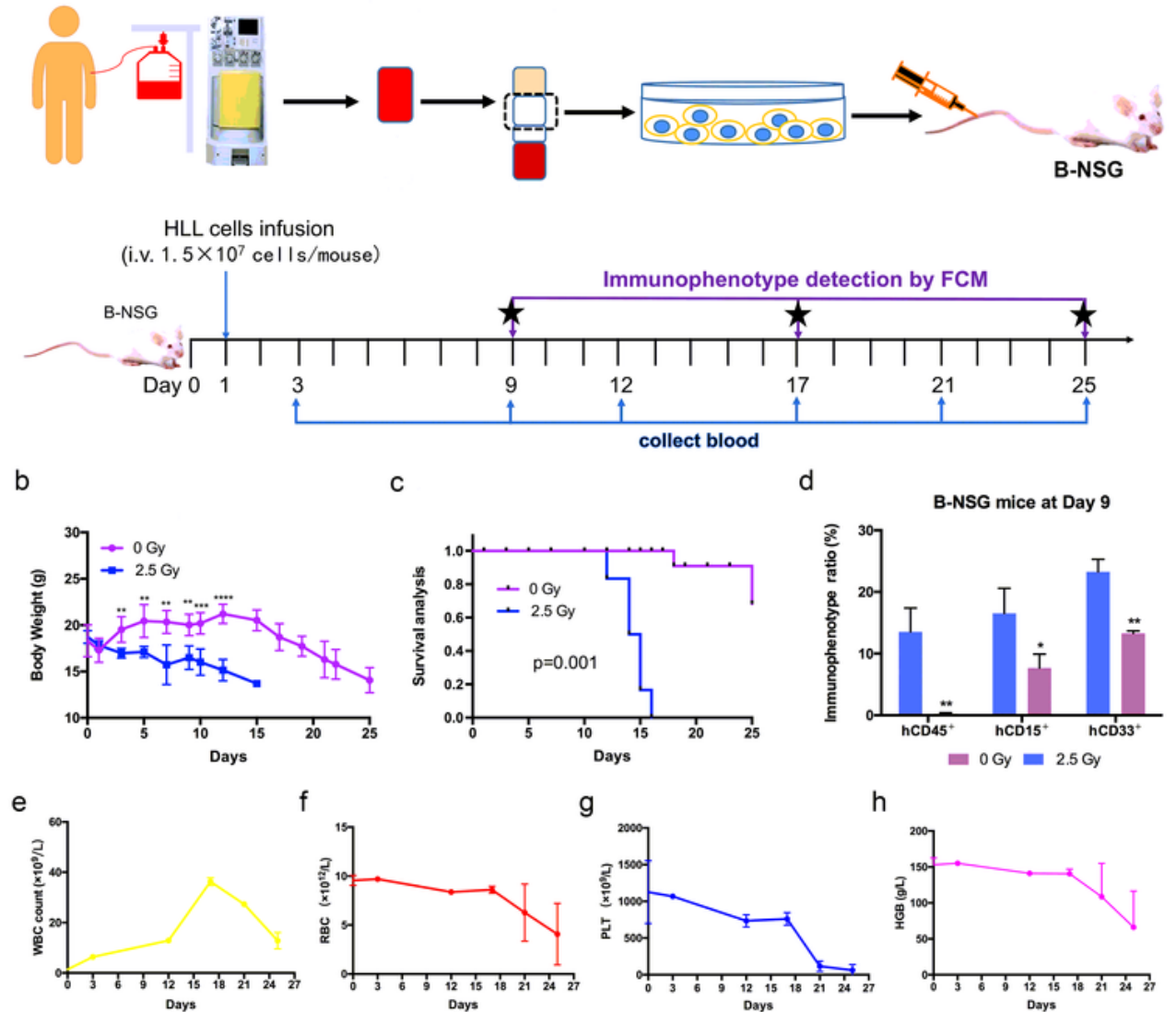

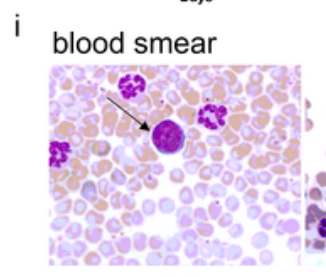

D9

$\mathrm{k}$ bone marrow

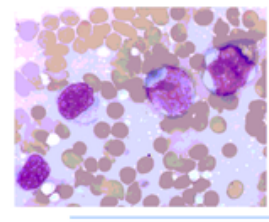

$\mathrm{m}$

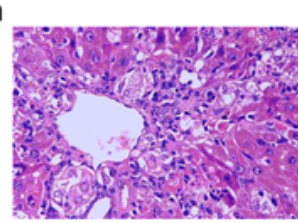

Liver $\mathrm{HE}$

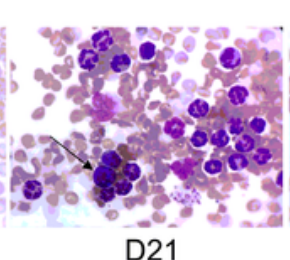

D21

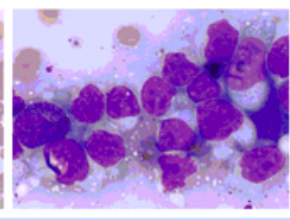

D25

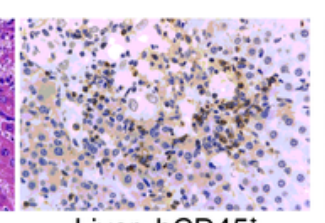

Liver hCD45 j

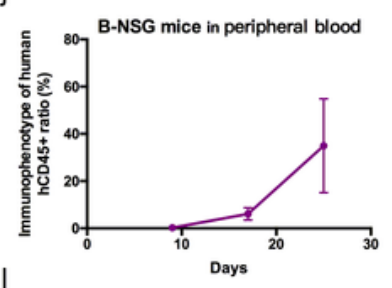

D25

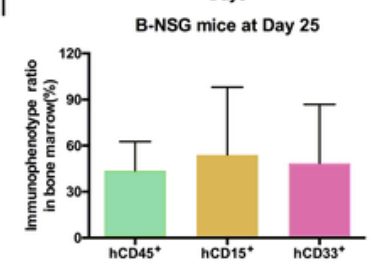

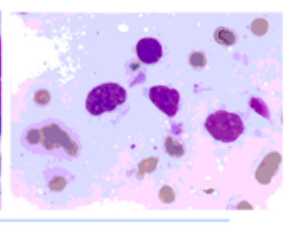

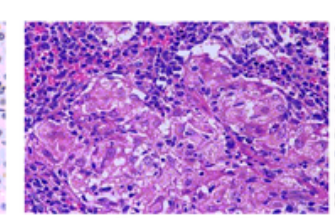

Spleen HE

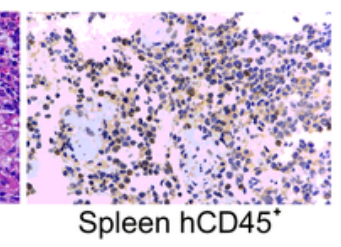

Figure 1

Establishment of the model of HLL with PBMCs of patients. a The HLL cells were collected from HLL patients using a Fresenius COM.TEC machine, and then the PBMCs were separated with a Ficoll kit and cultured. After irradiation at day 0, the B-NSG mice were treated with 0 Gy $(n=5)$ or $2.5 \mathrm{~Gy}(n=5)$ X-rays for total body irradiation and intravenously injected with harvested from PBMCs of human HLL cells from Patient \#1 mixed with granulocyte colony-stimulating factor. Every mouse was injected with $1.5 \times 107$ 
cells within $24 \mathrm{~h}$ of irradiation at day 1 . Blood samples were collected to test the WBC counts at D3, D9, D12, D17, D21 and D25. The immunophenotypes of leukemia cells, including hCD45+, hCD15+ and hCD33+, were detected by flow cytometry on days 9,7 , and 25 . b The body weights were analyzed using

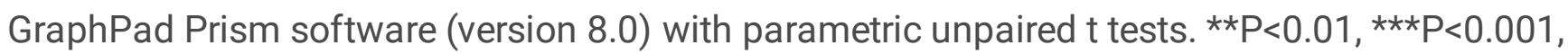

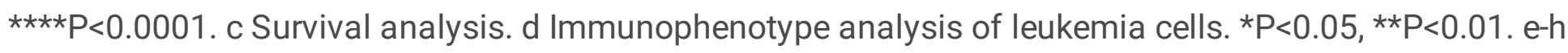
Routine blood tests with a Coulter STKS automated blood cell analyzer. e, WBC counts. f, RBC counts. g, PLT counts. h, HGB content. i Blood smear analysis were stained via Wright's staining and observed with a microscope under an oil immersion lens to measure the ratio of leukemia cells. $j$ Detection of the immunophenotype in peripheral blood by BD FACS Verse flow cytometry. HLL cells were stained with human anti-CD45-PerCPcy5.5, anti-CD15-FITC, and anti-CD33-PE antibodies. k Bone marrow smear analysis. I Detection of the immunophenotype in bone marrow by flow cytometry at day $25 \mathrm{~m}$ Histopathological analysis of leukemia cell infiltration into liver and spleen tissues. Magnification: $\times 400$. 
a

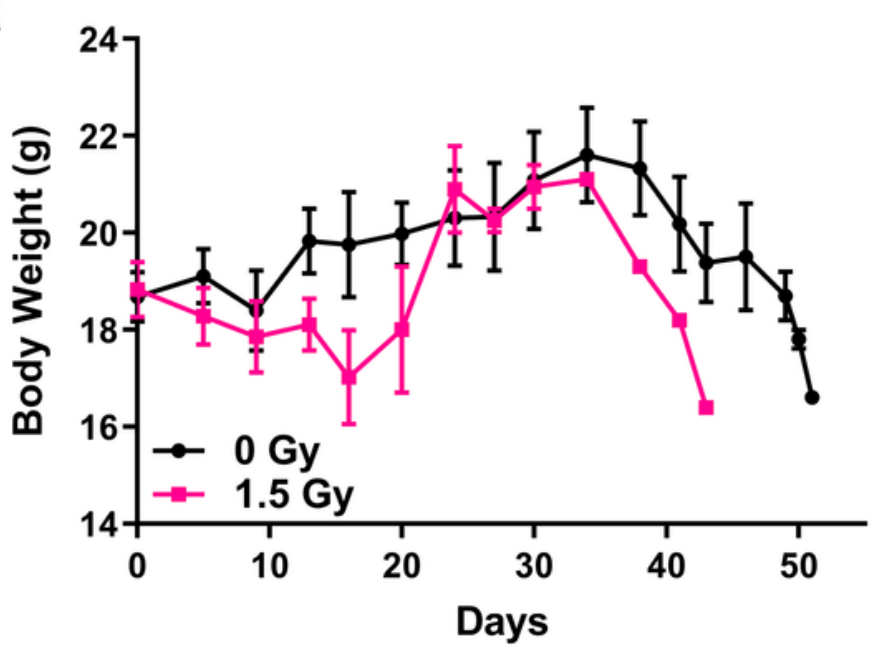

C

0 Gy in PB

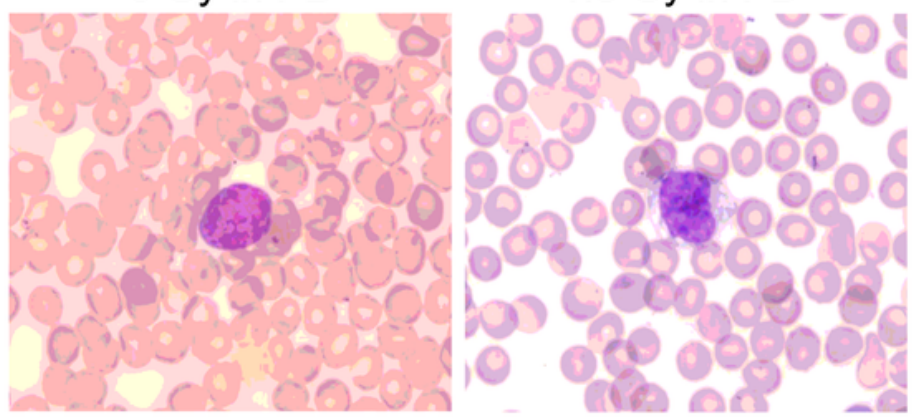

e

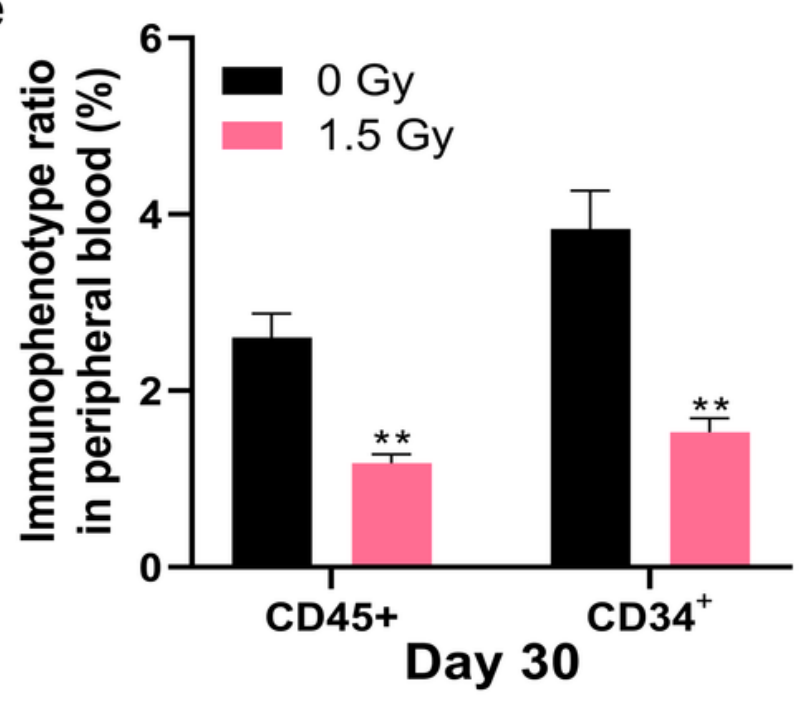

b

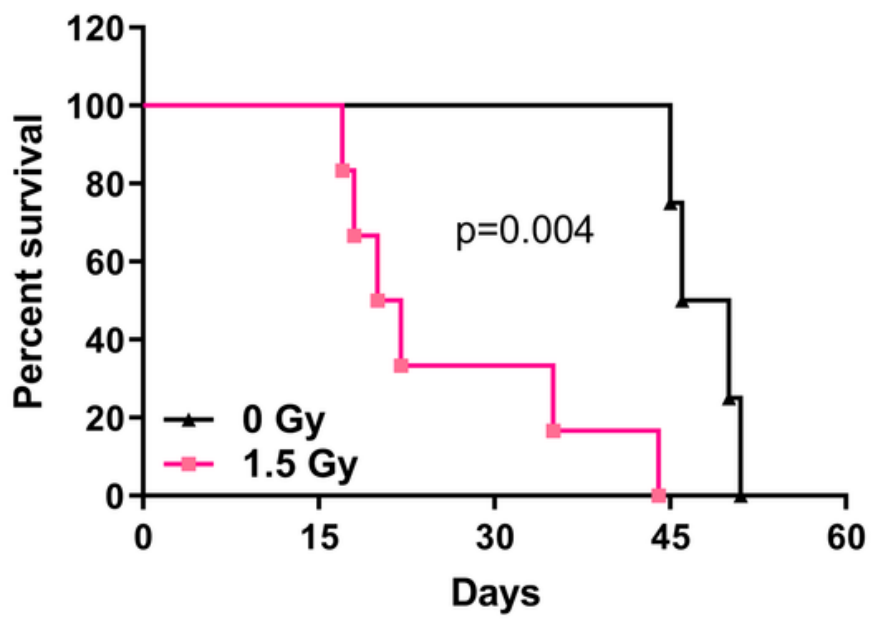

d

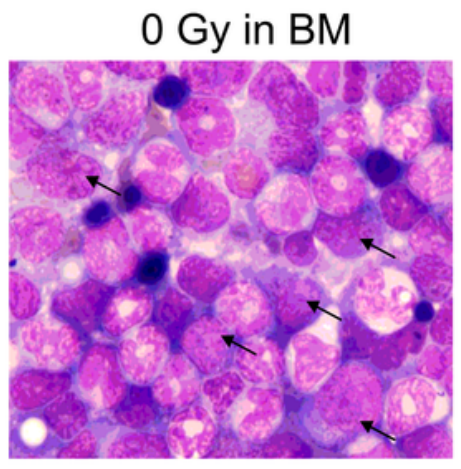

$1.5 \mathrm{~Gy}$ in BM

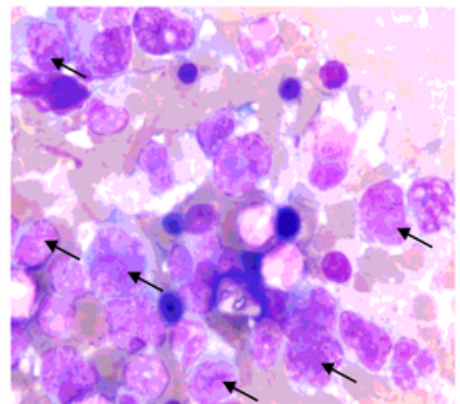

f

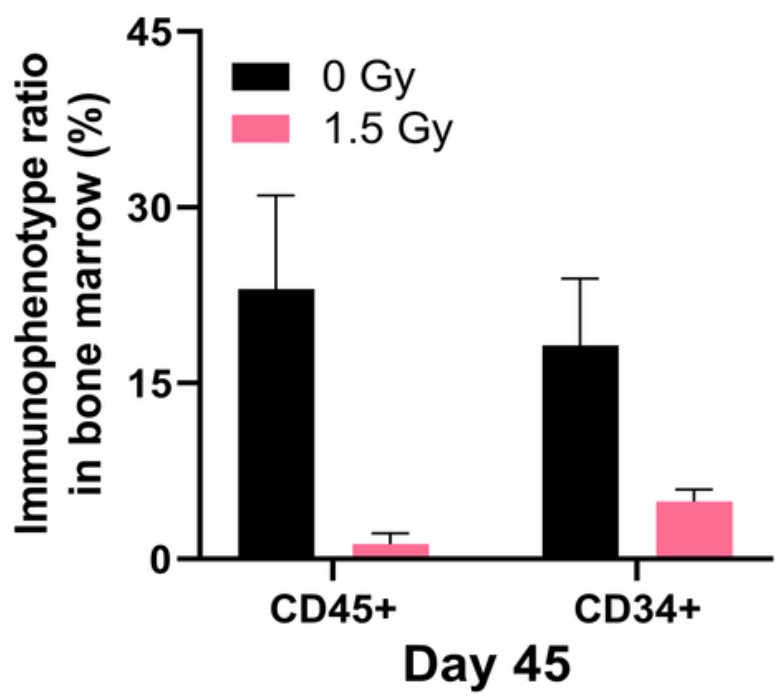

Figure 2

Construction of the HLL model with BM-derived cells of patients a On day 0, B-NSG mice were treated with $0 \mathrm{~Gy}(\mathrm{n}=5)$ or $1.5 \mathrm{~Gy}(\mathrm{n}=6)$ X-rays for total body irradiation and intravenously injected with harvested BM-derived cells from a human HLL patient (Patient \#3) at day 1 , then tested the change in body weight at intervals. b Survival analysis. c Blood smear analysis. PB, peripheral blood. $d$ Bone marrow smear analysis. BM, bone marrow. e Immunophenotype analysis of leukemia cells in peripheral blood at day 30 
by flow cytometry. ${ }^{\star \star} P<0.01$. $\mathrm{f}$ Detection of the immunophenotype in bone marrow by flow cytometry after mice were sacrificed.

a

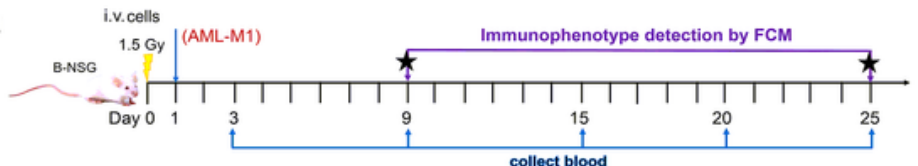

b

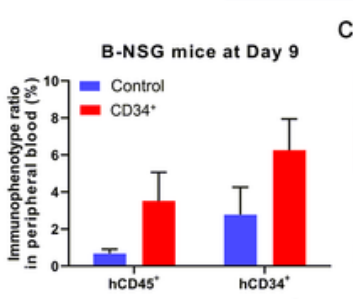

C

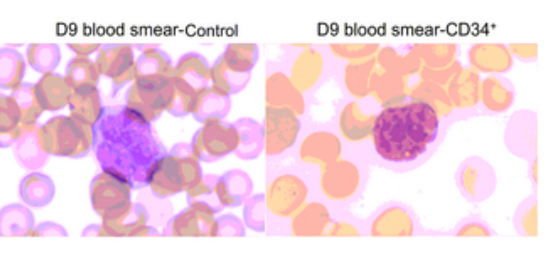

d
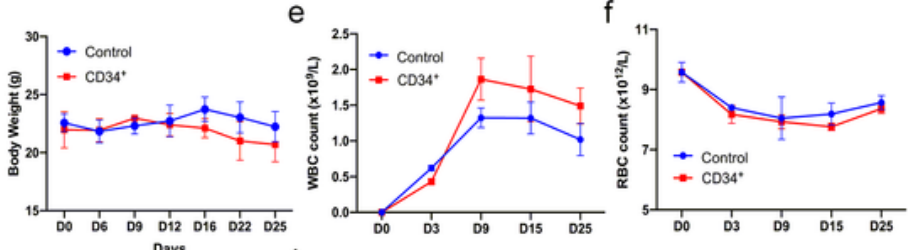

g

$\mathrm{h}$
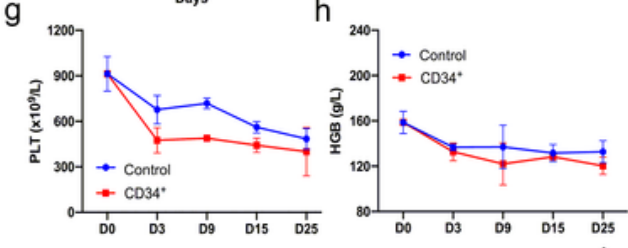

i 25 blood smear-controt

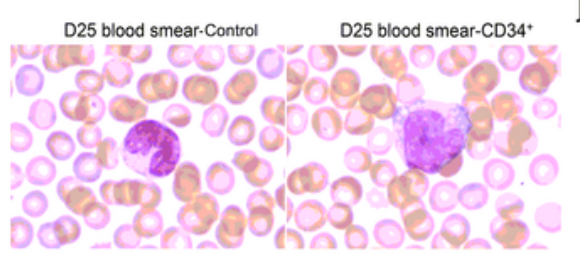

k
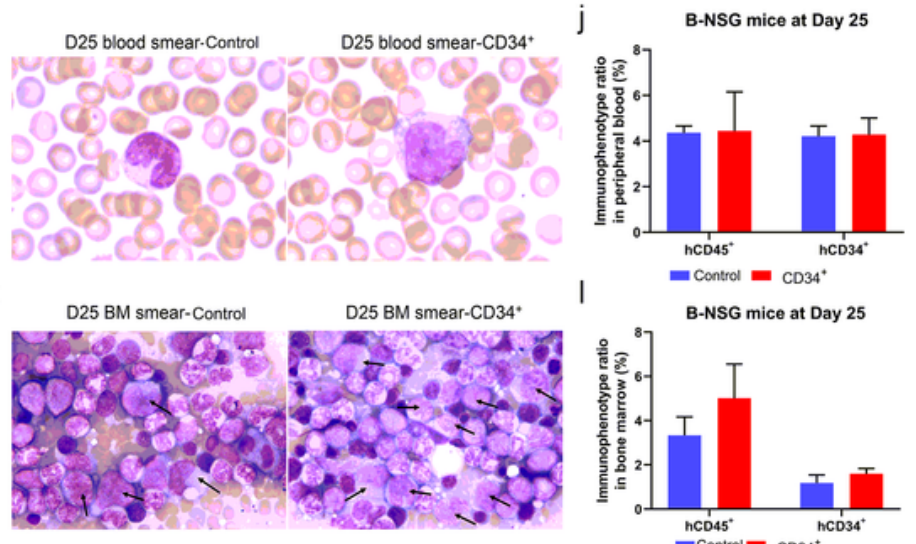

$\mathrm{m}$

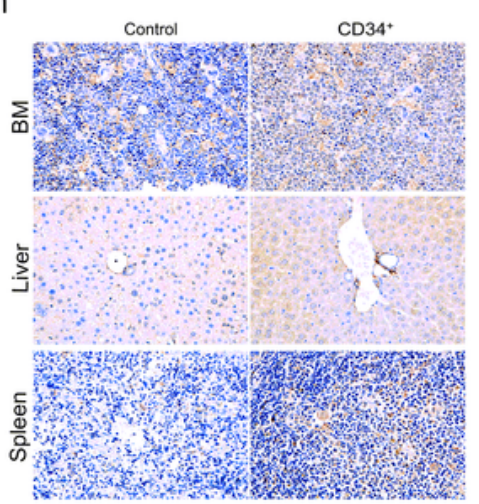

$\mathrm{n}$
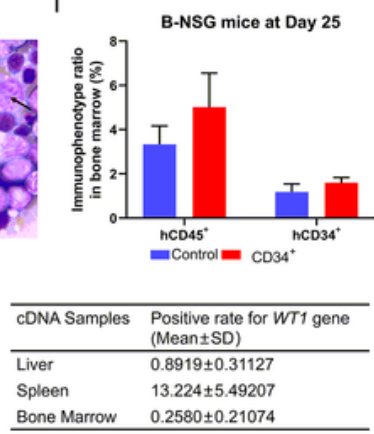

○

aacet ggt ggt ggt tggagcaggtggtgtt ggg

NRAS tetgaccaccaccaacctegtceaccacaacec

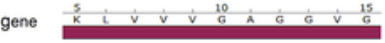

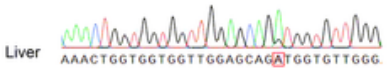

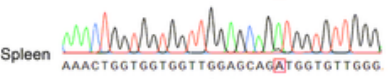

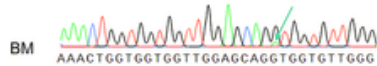

Figure 3

Construction of a CD34+ hematopoietic stem cell-derived xenograft model of HLL. a The workflow of model construction. The B-NSG mice were treated with $1.5 \mathrm{~Gy}$ X-rays for total body irradiation on day 0 and intravenously injected with isolated control cells $(n=5)$ or $1.5 \times 106$ CD34+ cells using magnetic 
beads $(\mathrm{n}=5)$ from Patient \#2 (WT1 and NRAS mutation) mixed with G-CSF within $24 \mathrm{~h}$ of irradiation. b Detection of immunophenotype in peripheral blood by flow cytometry at day 9. c Blood smear analysis at day 9. d The analysis of body weight. e-h Routine blood tests. e, WBC. f, RBC. g, PLT. h, HGB. i Blood smear analysis at day 25. j Detection of immunophenotype in peripheral blood at day 25. The cells were stained with human anti-CD45-PerCPcy5.5 and anti-CD34-APC antibodies. $\mathrm{k}$ Bone marrow smear analysis at day 25. I Detection of immunophenotype in bone marrow at day $25 . \mathrm{m}$ Immunohistochemical analysis of leukemia cell infiltration into bone marrow, liver and spleen tissues. The tissues were stained with antibodies against human anti-CD45+. BM, bone marrow. Magnification: $\times 400 . \mathrm{n}$ Detection of the positivity rate of the WT1 gene using a fluorescence quantitative PCR instrument in tissues of mice in the CD34+ group. o Detection of NRAS mutation in tissues of mice in the CD34+ group. Green represents base $A$, red represents base $T$, blue represents base $C$ and black represents base $G$. The green arrow represents a mutation in BM. BM, bone marrow. 


\begin{tabular}{|c|c|c|c|c|c|c|c|c|c|}
\hline $\begin{array}{l}\text { Patient- } \\
\text { derived } \\
\text { cells types }\end{array}$ & $\begin{array}{l}\text { Patient } \\
\text { survival }\end{array}$ & $\begin{array}{l}\text { Survival } \\
\text { months of } \\
\text { patient }\end{array}$ & $\begin{array}{l}\text { Patient } \\
\text { cells- } \\
\text { derived }\end{array}$ & $\begin{array}{l}\text { Gene mutation types } \\
\text { with patient cells }\end{array}$ & $\begin{array}{l}\text { Number } \\
\text { of mice }\end{array}$ & $\begin{array}{c}\text { X-ray } \\
\text { irradition at } \\
\text { DO }\end{array}$ & $\begin{array}{l}\text { Injection of } \\
\text { cell counts }\end{array}$ & $\begin{array}{l}\text { Survival } \\
\text { times when } \\
\text { sacrifice }\end{array}$ & Published/unpublished \\
\hline AML-M5 & death & 15 & PB & No mutation & 19 & $2.5 \mathrm{~Gy}$ & $1.7 \times 10^{7} /$ mouse HLL cells & 20 days & $\begin{array}{c}\text { J Exp Clin Cancer Res, } \\
\text { 2018. 37(1): } 277\end{array}$ \\
\hline AML-M5 & death & 10 & PB & NPM1, DNMT3A & 11 & $0 \mathrm{~Gy}$ & $1.5 \times 10^{7} /$ mouse HLL cells & 31 days & $\begin{array}{c}\text { Carbohydr Polym, } 2020 . \\
\text { 240: } 116329 .\end{array}$ \\
\hline AML-M5 & death & 20 & PB & WT1, DNMT3A, FLT3 & 16 & $1.5 \mathrm{~Gy}$ & $1.0 \times 10^{7} /$ mouse HLL cells & 21 days & $\begin{array}{l}\text { Oxid Med Cell Longev, } \\
\text { 2020. 2020, } 1295984 .\end{array}$ \\
\hline AML-M5 & death & 20 & PB & WT1, DNMT3A, FLT3 & 23 & $2.0 \mathrm{~Gy}$ & $2.0 \times 10^{7} /$ mouse HLL cells & 20 days & $\begin{array}{l}\text { Free radical bio med, } \\
\text { 2019. 146: } 275-286 \text {. }\end{array}$ \\
\hline AML-M5 & death & 10 & PB & $\begin{array}{l}\text { NRAS, NPM1, } \\
\text { DNMT3A }\end{array}$ & 5 & $2.5 \mathrm{~Gy}$ & $1.5 \times 10^{7} /$ mouse HLL cells & 17 days & Unpublished \\
\hline AML-M5 & death & 10 & PB & $\begin{array}{l}\text { NRAS, NPM1, } \\
\text { DNMT3A }\end{array}$ & 5 & 0 Gy & $1.5 \times 10^{7} /$ mouse HLL cells & 25 days & Unpublished \\
\hline AML-M1 & death & 17 & PB & WT1, NRAS & 5 & $1.5 \mathrm{~Gy}$ & $1.5 \times 10^{7} /$ mouse HLL cells & 25 days & Unpublished \\
\hline AML-M1 & death & 17 & PB & WT1, NRAS & 5 & $1.5 \mathrm{~Gy}$ & $1.5 \times 10^{6} /$ mouse CD $34+$ cells & 25 days & Unpublished \\
\hline AML-M1 & live & 2 & BM & WT1 & 6 & $1.5 \mathrm{~Gy}$ & $2 \times 10^{6} /$ mouse CD $34+$ cells & 44 days & Unpublished \\
\hline AML-M1 & live & 2 & $\mathrm{BM}$ & WT1 & 5 & 0 Gy & $1.5 \times 10^{7} /$ mouse HLL cells & 51 days & Unpublished \\
\hline AML-M5 & death & 20 & PB & WT1, DNMT3A, FLT3 & 28 & $1.5 \mathrm{~Gy}$ & $1.0 \times 10^{7} /$ mouse HLL cells & 31 days & Unpublished \\
\hline
\end{tabular}

b

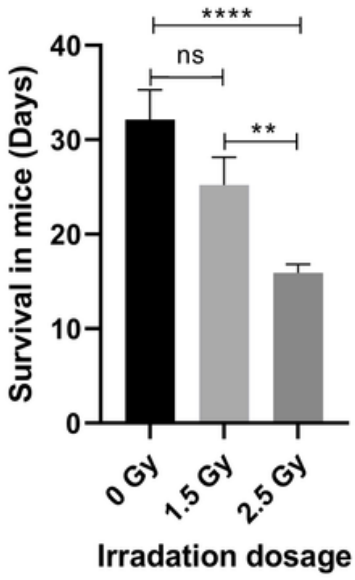

f
C

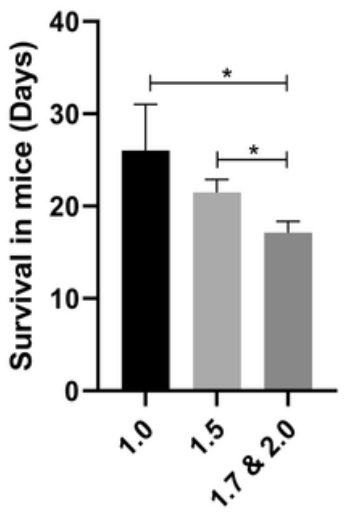

Injection HLL cells in $\mathrm{PB}\left(\times 10^{7} /\right.$ mouse $)$ d

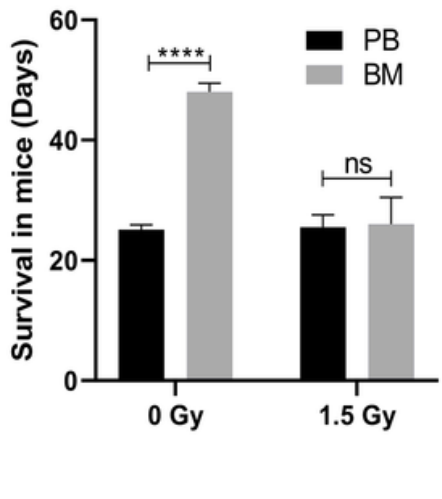

e

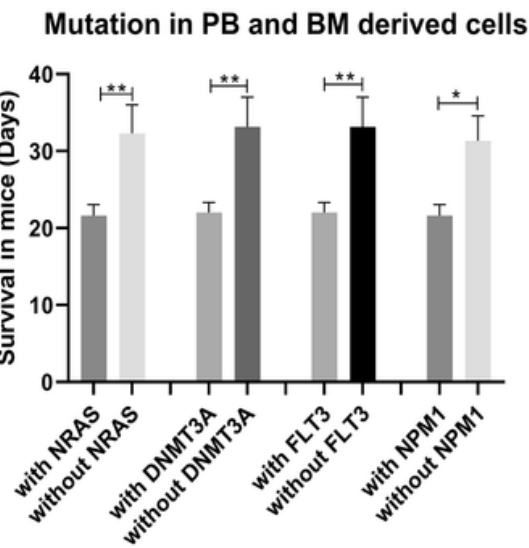

The correlation analysis between the survival time in mice and in patients

\begin{tabular}{lllllll}
\hline Item & Unstandardized B & $\mathbf{9 5 \%} \mathbf{C l}$ for B & $\begin{array}{l}\text { Standardized } \\
\text { Coefficient Beta }\end{array}$ & $\mathbf{t}$ & $\boldsymbol{P}$ value & $\mathbf{r}^{2}$ \\
\hline Survival time in mice & -0.456 & $-0.809 \sim-0.104$ & -0.726 & -2.987 & 0.017 & 0.527 \\
\hline
\end{tabular}

\section{Figure 4}

Summary of HLL models constructed with B-NSG mice. a List of experiments for HLL model establishment in B-NSG mice intravenously injected (i.v.) with patient-derived cells at D1. PB, peripheral blood. BM, bone marrow. The survival months were calculated from leukapheresis to presentation as of March 2021. b Analysis of the survival time in mice at different irradiation dosages. c Analysis of the survival time in mice with different injection cell counts from the PB of patients. $d$ Analysis of the survival time in mice injected with cells derived from $\mathrm{PB}$ and $\mathrm{BM}$ with irradiation of $0 \mathrm{~Gy}$ or $1.5 \mathrm{~Gy}$. e Analysis of 
survival time in mice with different gene mutations. $f$ Correlation analysis was performed to characterize the correlation between the survival time in mice and in patients by a linear regression model with variable selection "entered" with SPSS software (version 26.0). ${ }^{*} P<0.05,{ }^{\star} P P<0.01,{ }^{\star} * \star \star P<0.0001$. ns, no significance.

\section{Supplementary Files}

This is a list of supplementary files associated with this preprint. Click to download.

- AdditionalfileFigureS1.docx 